\title{
UV-Vis Absorption Study of cis- and trans-Poly(phenylacetylene)s Based on CNDO/S Calculation
}

\author{
Fumiaki IshiI ${ }^{*}{ }^{\dagger}$ Shigeyuki Matsunami,${ }^{\dagger \dagger}$ Minako Shibata, ${ }^{\dagger \dagger}$ \\ and Toyoji KaKUCHI \\ * Division of Applied Physics, Graduate School of Engineering, Hokkaido University, \\ Sapporo 060-8628, Japan \\ Division of Bioscience, Graduate School of Environmental Earth Science, Hokkaido University, \\ Sapporo 060-8628, Japan
}

(Received August 12, 1998)

\begin{abstract}
Before and after the thermal isomerization, the observed ultraviolet-visible (UV-Vis) absorptions of the phenyl groups and conjugated main chain for cis- and trans-poly(phenylacetylene)s (PPAs) in solution were investigated on the basis of CNDO/S calculation for the cis-transoidal and $\pm \tau^{\circ}$-deflected trans-transoidal structures. The two kinds of the UV-Vis absorption peaks were observed at 263 and $390 \mathrm{~nm}$ for the cis-transoidal PPA and at 227 and $248 \mathrm{~nm}$ for $90^{\circ}$-deflected trans-transoidal PPA, which were due to the $\pi-\pi^{*}$ transitions of phenyl groups and the conjugated bonds in the main chain. After cis-trans isomerization, the larger blue shift of the $\pi-\pi^{*}$ transition absorption in the UV spectrum was confirmed from the $\mathrm{CNDO} / \mathrm{S}$ calculation for the $\pm 90^{\circ}$-deflected trans-transoidal structures.
\end{abstract}

KEY WORDS UV-Vis Absorption / cis- and trans-Poly(phenylacetylene)s / CNDO/S Calculation /

Recently, substituted poly(phenylacetylene) (PPA) have been paid attention as polymeric materials possessing magnetic and nonlinear optical properties. ${ }^{1}$ The properties and behaviors of the nonlinear magnetic susceptibilities are characterized by the underlying $\pi$-electron and its excitation process in the conjugated bonds, which depend on the molecular chain structures of cis-transoidal, trans-cisoidal, and trans-transoidal forms. However, the thermal isomerization and the chain structures of PPA were not verified largely up to date, because the IR and high resolution NMR spectra after thermal isomerization were complex and broad. ${ }^{2-4}$

In a series of NMR studies, ${ }^{5,9}$ we have clarified first that chain structures of PPA in the solid state are cis-transoidal and $\pm 80^{\circ}$ deflected trans-transoidal before and after DSC exotherm at about $180^{\circ} \mathrm{C}$, respectively, and then the exothermic reaction is the cistrans isomerization. Especially, it is worth noting that the ${ }^{13} \mathrm{C}$ peak of main chain carbons in the ${ }^{13} \mathrm{C}$ NMR spectrum in the solid state for each polymer shifted to upfield by $2-3 \mathrm{ppm}$ after cis-trans isomerization, in contrast to its downfield shift for polyacetylene. ${ }^{9}$ This upfield shift was attributed to the greater electron density on the main chain carbons in the $\tau=80^{\circ}$ deflected angle trans-transoidal chain.

It is well-known that the UV-Vis absorption of conjugated polyene as poly(acetylene) (PA) is very dependent on the stereochemical configuration and the effective conjugated length of the main chain. The UV absorption band of the $\pi-\pi^{*}$ transition for the transtransoidal configuration are located in a higher wavelength region than that for the cis-transoidal one. The wavelength $\lambda$ of absorption becomes greater as the length of the planar conjugated chain increases and the intensity also increases. In the UV-Vis spectra of PPA solutions, two kinds of the $\pi-\pi^{*}$ absorption peaks due to the conjugated double bonds of main chain and phenyl group have been observed in the wavelength region between 100 and $1000 \mathrm{~nm}$, as well as polyenes. However, after cis-trans isomerization, the corresponding UV-Vis absorption peaks for annealed PPA sample and their theoretical analyses have apparently not been reported to date.

In this paper, the assignment of observed UV-Vis absorption peaks and the wavelength $\lambda$ shift of absorption after cis-trans isomerization for PPA are investigated on the basis of UV theory within semiempirical molecular orbital CNDO/S calculation. ${ }^{10}$ The localization of the $\pi$-electrons in the conjugated main chain and its UV blue shift due to the deflected transtransoidal form will be discussed.

\section{EXPERIMENTAL}

\section{Sample}

cis- and trans-Samples of PPA were prepared by the same method described in previous papers. ${ }^{5,7,11}$ Number-average molecular weight and molecular weight distribution were 25000 and 1.76 , respectively, for cisPPA and those were 2500 and 1.33 , respectively, for trans-PPA.

\section{UV-Vis Measurements}

The UV-Vis spectra of PPA were run on a Hitachi U-3200 spectrophotometer with $10 \mathrm{~mm}$ glass cell in $\mathrm{CHCl}_{3}$ at room temperature.

\section{UV-Vis Absorption Band Calculations}

UV-Vis absorption bands of five conformers for PPA were theoretically calculated using $\mathrm{CNDO} / \mathrm{S}^{10}$ with MOS-F of Anchor II software (Fujitsu) on a Fujitsu $\mathrm{S}-4 / 5$ workstation. The same chain structures of the five

\footnotetext{
${ }^{\dagger}$ To whom all correspondence should be addressed (Tel: +81+11-706-6642, Fax: +81+11-716-6175, E-mail: fishii@eng.hokudai.ac.jp).

${ }^{\dagger \dagger}$ Research Fellowship of the Japan Society for the Promotion of Science.
} 


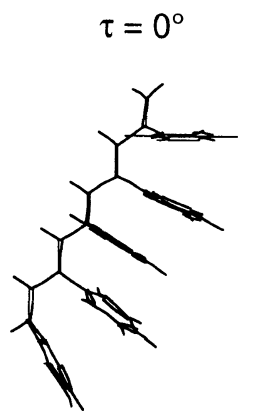

trans-transoidal $\tau=60^{\circ}$

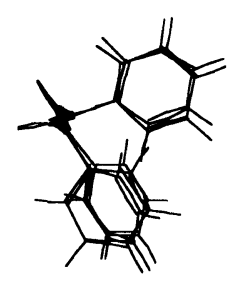

deflected trans-transoidal $\tau=120^{\circ}$

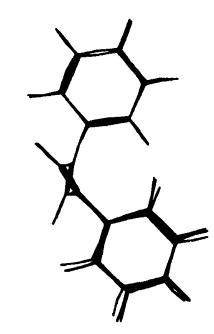

deflected trans-transoidal $\tau=180^{\circ}$

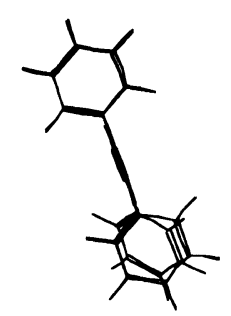

cis-transoidal

Figure 1. The back-bone geometry of the deflected trans chain for five confomer of PPA projected on the perpendicular plane to its axis. The deflected angle of $\tau$ corresponds to the dihedral angle between two double bonds in the main chain.

conformers as cis- and trans-forms determined by AM1 in previous papers were used in the UV transition band, which were trans-transoidal, trans-cisoidal, cis-transoidal, and deflected trans-transoidal forms. Although the formation energy of the $80^{\circ}$-deflected trans-transoidal form was minimal, the theoretical bands of the $\pi-\pi^{*}$ transitions of $\left(\varphi_{96} \rightarrow \varphi_{99}\right)$ and $\left(\varphi_{96} \rightarrow \varphi_{97}\right)$ for the phenyl group and the main chain for deflected trans-transoidal form were fitted in the observed two-peaks as a function of the deflected angle $\tau$ using the least square method, and then the other theoretical UV absorption bands were optimized. The helical structure of the chain was not used in the UV calculation because the steric hindrance of the phenyl groups in PPA chain due to the Van der Waals interaction between the side and main chains allowed the $\pm \tau$-deflected trans-transoidal conformation, but did not allow the considerable helical structures of the chain. ${ }^{7,9}$

Figure 1 shows the back-bone structures of the same deflected trans chain conformation projected on a perpendicular plane to its axis. The deflected angle of $\pm \tau$ corresponds to the \pm dihedral angle between two double bonds in the main chain. The optimal geometry of various trans-transoidal forms against the deflected angle $\tau$ was calculated in $10^{\circ}$ interval from $\tau=0^{\circ}$ (trans-transoidal) to $180^{\circ}$ (trans-cisoidal and cistransoidal). The electron charge density of PPA was calculated for optimal conformations in the fiveconformer as a function of $\tau$.

\section{RESULTS AND DISCUSSION}

Figure 2 shows the UV-Vis spectra of cis- and deflected trans-PPA in $\mathrm{CHCl}_{3}$ in solution, respectively. Solid and dotted lines are the UV-Vis absorption of cis and deflected trans-PPAs, respectively. In the UV spectrum of cis-PPA, there are a broad absorption peak at $263 \mathrm{~nm}$ and a plateau peak shoulder at about $390 \mathrm{~nm}$ between 300 and $600 \mathrm{~nm}$. On the other hand, there are a sharp absorption peak at $227 \mathrm{~nm}$ and a broad peak with the skirts at $248 \mathrm{~nm}$ in the UV spectrum of deflected trans-PPA.

Here, we will theoretically calculate the UV-Vis absorption bands of five conformers for PPA using $\mathrm{CNDO} / \mathrm{S} .{ }^{10}$ The chain structures of the five conformers in the $\pi-\pi^{*}$ transition calculation are the trans-transoidal,

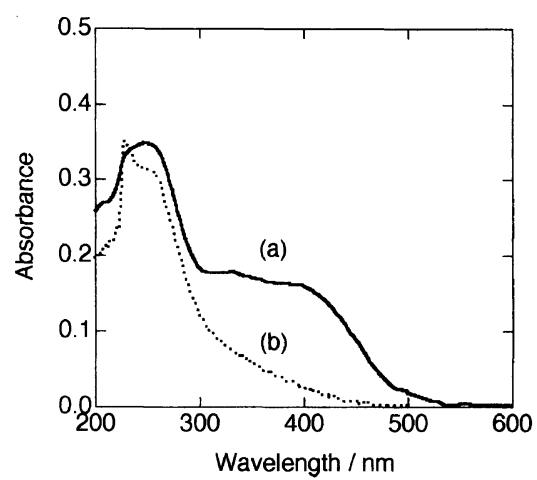

Figure 2. UV-Vis spectra of (a) cis-PPA and (b) deflected trans-PPA in $\mathrm{CHCl}_{3}$ solution at room temperature.

trans-cisoidal, cis-transoidal, and $\pm \tau$-deflected transtransoidal conformations in an interval of deflected angle $\tau=10^{\circ}$, as well as those used in previous reports. ${ }^{7,9}$

In the SCF-MO calculation, ${ }^{12}$ we used the configuration interaction (CI) method to evaluate the most probable wave function for the true excitation and basis states of $\pi-\pi^{*}$ transition, because the respective electron configurations mix in the excitation states, which are restricted between the five highest occupied molecular orbitals and the five lowest unoccupied molecular ones. The molecular orbitals of the five conformers for PPA consisted of 192 basis sets and 192 valence electrons occupying up to the 96-th molecular orbital, and the two-center integrals were estimated based on NishimotoMataga equation. ${ }^{13}$

Figure 3 shows the schematic representation of the orbital energy diagram from $\varphi_{92}$ to $\varphi_{101}$ for cistransoidal and $90^{\circ}$-deflected trans-transoidal PPAs calculated theoretically. The obtained orbital energies of $\varphi_{96}(\mathrm{HOMO})$ and $\varphi_{97}$ (LUMO) are $E\left(\varphi_{96}\right)=-7.664$ $\mathrm{kcal} \mathrm{mol}^{-1}$ and $E\left(\varphi_{97}\right)=-2.011 \mathrm{kcal} \mathrm{mol}^{-1}$ for cistransoidal PPA, and $E\left(\varphi_{96}\right)=-8.319 \mathrm{kcal} \mathrm{mol}^{-1}$ and $E\left(\varphi_{97}\right)=-1.053 \mathrm{kcal} \mathrm{mol}^{-1}$ for deflected trans PPA. Energies for other occupied orbitals from $\varphi_{91}$ to $\varphi_{94}$ for deflected trans-transoidal PPA are higher than those for cis-transoidal PPA and tend to degenerate in energy, while only the energy level of LUMO shows substantial increases in energy among the unoccupied molecular orbitals.

Tables I and II list the calculated transition wavelengths, oscillator strengths, excited molecular orbitals, 


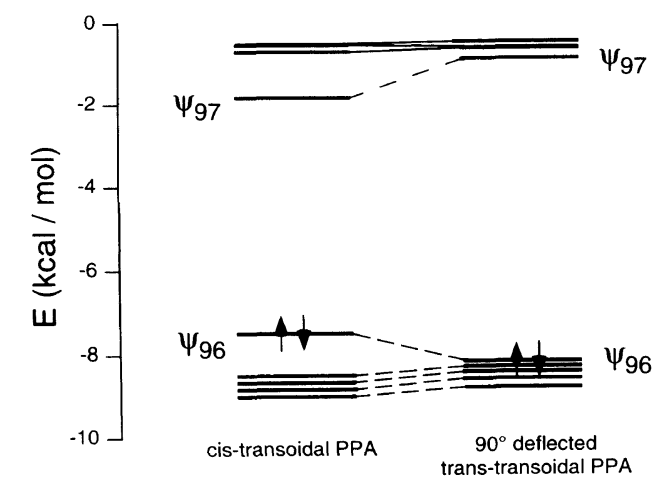

Figure 3. Schematic representation of the orbital energy diagram for cis-transoidal PPA and $90^{\circ}$-deflected trans-transoidal PPA calculated by $\mathrm{CNDO} / \mathrm{S}$.

and the $\mathrm{CI}$ compositions which represent the relative contribution of the transition. The resultant CI compositions for excited molecular orbitals indicate that the main contribution of $98 \%$ at $371.38 \mathrm{~nm}$ for cistransoidal PPA, which is attributed to the absorption of the main chain, come from the transition between $\varphi_{96}$ and $\varphi_{97}$, and the contribution of $84 \%$ at $257.93 \mathrm{~nm}$ is due to the side chain, mainly the transition between $\varphi_{96}$ and $\varphi_{99}$. On the other hand, the absorption bands of the main and side chains for the deflected trans PPA shift to 275.14 and $248.88 \mathrm{~nm}$ with decreases in CI compositions from 98 to $69 \%$ and from 84 to $49 \%$, respectively.

Comparisons between the experimental and theoretical UV-Vis spectra of for cis-PPA and $90^{\circ}$ deflected transPPA are shown in figure 4. In each figure, discrete solid lines are the theoretical UV absorption on the basis of UV calculation within $\mathrm{CNDO} / \mathrm{S}$ and the solid line is the experimental absorption data. The theoretical absorption lines of all bands for cis- and deflected trans-transoidal were optimized.

In Figure $4 \mathrm{a}$, the calculated absorption lines at 257.93 and $371.38 \mathrm{~nm}$ for cis-transoidal form are due to the $\pi-\pi^{*}$ transitions of $\left(\varphi_{96} \rightarrow \varphi_{99}\right)$ and $\left(\varphi_{96} \rightarrow \varphi_{97}\right)$ for the phenyl group and the conjugated main chain between HOMO and LUMO along their axis direction. They are in good agreement with the wavelengths of 263 and $390 \mathrm{~nm}$ in the observed UV-Vis spectrum, respectively, as well as the theoretical wavelengths of further bands. This result indicates that the experimental broad and plateau shoulder peaks are due to the $\pi-\pi^{*}$ transition for the phenyl groups and the conjugated main chain, respectively. In Figure 4b, the theoretical UV absorption solid lines of the $90^{\circ}$-deflected trans-transoidal chain for PPA localizes in the region between 230 and $300 \mathrm{~nm}$ of the experimental spectrum of the solid line. They are in greater agreement with the experimental UV spectra, compared with theoretical result of the $80^{\circ}$-deflected trans-transoidal five conformers as shown in Figure 4c. The solid lines at 248.88 and $275.14 \mathrm{~nm}$ due to the $\pi-\pi^{*}$ absorption bands of the phenyl groups and the conjugated main-chain for the $90^{\circ}$ deflected transtransoidal forms agree with the board absorption peaks at 227 and $248 \mathrm{~nm}$ in the observed UV spectrum of the solid line. Then, these observed peaks come from the $\pi-\pi^{*}$ absorption bands of the phenyl groups and the conjugated main-chain, respectively.
Table I. Wavelength, oscillator strength, energy levels of molecular orbitals, and CI composition for cis-transoidal PPA calculated by $\mathrm{CNDO} / \mathrm{S}$

\begin{tabular}{|c|c|c|c|c|}
\hline State & $\begin{array}{c}\text { Wave length/ } \\
\mathrm{nm}\end{array}$ & $\begin{array}{l}\text { Oscillator } \\
\text { strength }\end{array}$ & $\mathrm{MO}^{\mathrm{a}}$ & $\begin{array}{c}\text { CI coeff. } y \\
\%\end{array}$ \\
\hline 1 & 371.38 & 1.746 & $96 \rightarrow 97$ & 98 \\
\hline \multirow[t]{2}{*}{2} & 317.31 & 0.001 & $94 \rightarrow 97$ & 55 \\
\hline & & & $95 \rightarrow 97$ & 40 \\
\hline \multirow[t]{3}{*}{3} & 305.47 & 0.006 & $93 \rightarrow 97$ & 41 \\
\hline & & & $95 \rightarrow 97$ & 37 \\
\hline & & & $94 \rightarrow 97$ & 18 \\
\hline \multirow[t]{3}{*}{4} & 295.01 & 0.001 & $93 \rightarrow 97$ & 53 \\
\hline & & & $94 \rightarrow 97$ & 24 \\
\hline & & & $95 \rightarrow 97$ & 17 \\
\hline \multirow[t]{2}{*}{5} & 275.28 & 0.024 & $96 \rightarrow 98$ & 41 \\
\hline & & & $92 \rightarrow 97$ & 21 \\
\hline \multirow[t]{2}{*}{6} & 266.30 & 0.011 & $96 \rightarrow 101$ & 78 \\
\hline & & & $92 \rightarrow 101$ & 5 \\
\hline 7 & 257.93 & 0.004 & $96 \rightarrow 99$ & 84 \\
\hline \multirow[t]{2}{*}{8} & 256.81 & 0.034 & $92 \rightarrow 97$ & 69 \\
\hline & & & $96 \rightarrow 98$ & 23 \\
\hline 9 & 240.12 & 0.007 & $96 \rightarrow 100$ & 83 \\
\hline \multirow[t]{2}{*}{10} & 225.56 & 0.009 & $94 \rightarrow 98$ & 54 \\
\hline & & & $93 \rightarrow 98$ & 31 \\
\hline
\end{tabular}

a The 96-th and 97-th molecular orbitals corresponded to HOMO and LUMO, respectively. ${ }^{b}$ The relative contribution of UV transition.

Table II. Wavelength, oscillator strength, energy levels of transition molecular orbitals, and CI composition for $90^{\circ}$-deflected trans-transoidal PPA calculated by $\mathrm{CNDO} / \mathrm{S}$

\begin{tabular}{|c|c|c|c|c|}
\hline State & $\begin{array}{l}\text { Wave length/ } \\
\mathrm{nm}\end{array}$ & $\begin{array}{l}\text { Oscillator } \\
\text { strength }\end{array}$ & $\mathrm{MO}^{\mathrm{a}}$ & $\begin{array}{c}\text { CI coeff. }{ }^{b} / \\
\%\end{array}$ \\
\hline \multirow[t]{2}{*}{1} & 292.58 & 0.063 & $96 \rightarrow 98$ & 58 \\
\hline & & & $96 \rightarrow 100$ & 26 \\
\hline \multirow[t]{2}{*}{2} & 275.14 & 0.020 & $96 \rightarrow 97$ & 69 \\
\hline & & & $95 \rightarrow 97$ & 18 \\
\hline \multirow[t]{2}{*}{3} & 268.87 & 0.008 & $95 \rightarrow 97$ & 62 \\
\hline & & & $96 \rightarrow 97$ & 26 \\
\hline \multirow[t]{3}{*}{4} & 259.25 & 0.142 & $96 \rightarrow 100$ & 28 \\
\hline & & & $96 \rightarrow 98$ & 27 \\
\hline & & & $96 \rightarrow 99$ & 25 \\
\hline 5 & 255.02 & 0.021 & $95 \rightarrow 98$ & 73 \\
\hline \multirow[t]{3}{*}{6} & 248.88 & 0.030 & $96 \rightarrow 99$ & 49 \\
\hline & & & $96 \rightarrow 100$ & 21 \\
\hline & & & $95 \rightarrow 100$ & 15 \\
\hline \multirow[t]{2}{*}{7} & 247.27 & 0.223 & $94 \rightarrow 98$ & 19 \\
\hline & & & $95 \rightarrow 99$ & 17 \\
\hline \multirow[t]{2}{*}{8} & 237.07 & 0.009 & $95 \rightarrow 100$ & 36 \\
\hline & & & $94 \rightarrow 98$ & 32 \\
\hline \multirow[t]{2}{*}{9} & 235.50 & 0.231 & $94 \rightarrow 97$ & 49 \\
\hline & & & $94 \rightarrow 98$ & 35 \\
\hline \multirow[t]{3}{*}{10} & 230.02 & 0.047 & $94 \rightarrow 97$ & 24 \\
\hline & & & $94 \rightarrow 99$ & 20 \\
\hline & & & $94 \rightarrow 100$ & 18 \\
\hline
\end{tabular}

a The 96-th and 97-th molecular orbitals corresponded to HOMO and LUMO, respectively. ${ }^{\mathbf{b}}$ The relative contribution of UV transition.

Compared with the results of cis-PPA, the absorption peaks of the phenyl group and the conjugated main chain in the observed spectrum shift to lower wavelength by $35 \mathrm{~nm}$ from 263 to $227 \mathrm{~nm}$ and by $142 \mathrm{~nm}$ from 390 to $248 \mathrm{~nm}$, respectively, and theoretical ones by $9.05 \mathrm{~nm}$ from 257.93 to $248.88 \mathrm{~nm}$ and by $96.24 \mathrm{~nm}$ from 371.38 to $275.14 \mathrm{~nm}$, respectively. There is in qualitative agreement between theory and experiment on two absorp- 

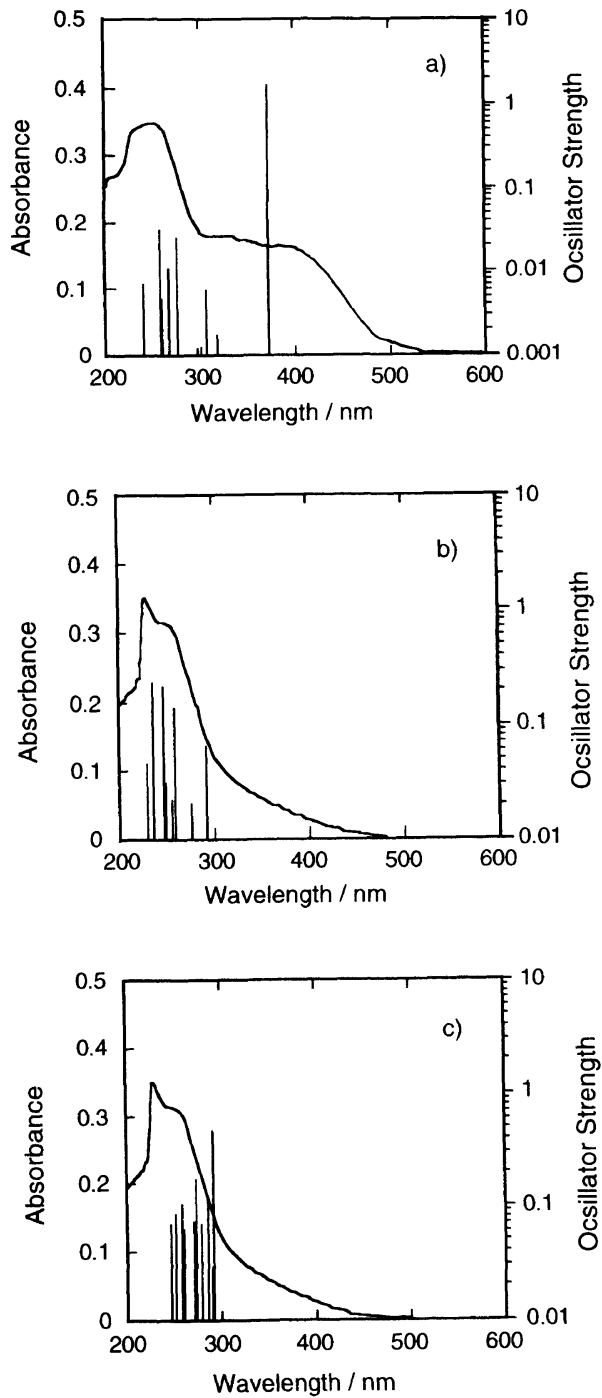

Figure 4. The comparison between experimental and theoretical UV-Vis spectra. Discrete solid lines were theoretical UV-Vis absorption bands for cis-transoidal PPA (a), $90^{\circ}$-deflected trans-transoidal PPA (b), and $80^{\circ}$-deflected trans-transoidal PPA calculated using CNDO/S (c).

tion peaks and lower wavelength shift of each peak after thermal isomerization. Thus, the $90^{\circ}$-deflected transtransoidal structure gives the larger energy gap between HOMO-LUMO for phenyl group and main chain, so that the respective $\pi-\pi^{*}$ transitions take place in a lower wavelength region, called as blue shift. In Figure 2, the disappearance of the absorption band around $390 \mathrm{~nm}$ in the observed UV-Vis spectrum for transPPA is caused by the blue shift of the conjugated main chain due to $90^{\circ}$ deflected trans-transoidal structure.

In previous papers, ${ }^{5-9}$ we reported from ${ }^{2} \mathrm{H}$ and ${ }^{13} \mathrm{C}$ NMR analyses of PPA, based on AM1 molecular orbital method, that after cis-trans isomerization the $80^{\circ}$ deflected trans-transoidal conformation of the chain is stable in solid state. Using PM3 approximation in comparison between theory and experiment for the ${ }^{13} \mathrm{C}$ NMR chemical shifts of the deflected trans PPA, the chain conformation was determined to be $75^{\circ}$-deflected trans-transoidal form. The determination of the deflected angle depended slightly on the semiemprical molecular orbital methods. As shown in Figure 4a, the observed UV spectrum of solid line does not agree with the theoretical one for the $80^{\circ}$-deflected trans-transoidal conformation in solid state. The deflected angle of the trans chain conformation fitted theoretically in Figure 5 increases by $10^{\circ}$ in solution. It is considered that the increase of the deflected angle may be due to an oscillational rotation of the chain about the single bond in solution.

On the other hand, we calculated the electron densities of $\mathrm{CH}$ and tertiary carbons, referred to as $\mathrm{C} 1$ and $\mathrm{C} 2$ carbons, respectively, in the conjugated main chain for the five conformers of deflected trans-PPA. The electron density of $\mathrm{C} 1$ carbon decreased from 4.122 to 4.111 with increasing of the deflected angle and that of the tertiary carbon increased from 4.016 to 4.046 . At $\tau=90^{\circ}$ the electron densities of $\mathrm{C} 1$ and $\mathrm{C} 2$ are 4.113 and 4.036, respectively.

These experimental and theoretical facts for PPA indicate that the deflected trans-transoidal conformation after thermal isomerzation gives rise to the localization of $\pi$-electrons in the conjugated main chain, i.e., characteristic blue shift of the $\pi-\pi^{*}$ transition absorption in the UV spectrum. We will report theoretically elsewhere in near future how the disagreement of $10^{\circ}$ is due to the CNDO/S and AM1 calculation approximations or molecular motions in solution.

\section{CONCLUSIONS}

The UV-Vis spectra of cis- and deflected transtransoidal forms for PPA in solution were investigated on the basis of UV-Vis theory within semiempirical molecular orbital method of $\mathrm{CNDO} / \mathrm{S}$. The observed UV spectrum for cis-PPA consisted of two broadened and shoulder peaks at 263 and about $390 \mathrm{~nm}$, and for deflected trans-PPA a sharp peak and broad one with skirts at 227 and $248 \mathrm{~nm}$. The two kinds of UV absorption peaks for each sample were due to the $\pi-\pi^{*}$ transitions of the phenyl groups and conjugated main chain. After cis-trans isomerization, the larger blue shift of the $\pi-\pi^{*}$ transition absorption for the conjugated main chain in the UV spectrum came from the localization of $\pi$-electrons due to the $90^{\circ}$-deflected structure of the trans-transoidal main chain. The increase of the deflected angle from $80^{\circ}$ in the solid to $90^{\circ}$ in solution may be attributed to an oscillational rotation of the chain about the single bond in solution.

\section{REFERENCES}

1. M. Buchmeiser and R. R. Schrock, Macromolecules, 28, 6642 (1995).

2. A. A. Berlin, M. I. Cherkashin, I. P. Chernysheva, Y. G. Aseyev, Y. I. Barkan, and P. P. Kisilitsa, Vysokomol. Soyedin, 9, 1840 (1967).

3. C. I. Simionescu and V. Percec, J. Polym. Sci., Polym. Chem. Ed., 18, 147 (1980).

4. A. Furlani, C. Napoletano, M. V. Russo, and W. J. Feast, Polym. Bull., 16, 311 (1986).

5. T. Kakuchi, S. Matsunami, H. Kamimura, F. Ishii, T. Uesaka, and K. Yokota, J. Polym. Sci., Part A, Polym. Chem., 33, 1431 (1995).

6. T. Kakuchi, S. Matsunami, H. Kamimura, and F. Ishii, J. Polym. Sci., Part B, Polym. Phys., 33, 2151 (1995).

7. S. Matsunami, T. Kakuchi, and F. Ishii, Macromolecules, 30, 1074 (1997). 
8. T. Kakuchi, S. Matsunami, K. Tsuda, and F. Ishii, Polym. Bull. 40, 533 (1998).

9. F, Ishii, T. Kakuchi, and S. Matsunami, submitted to J. Polym. Sci., Part B, Polym. Phys.

10. J. D. Bene and H. H. Jaffe, J. Chem. Phys., 48, 4050 (1968)
11. S. Matsunami, T. Watanabe, H. Kamimura, T. Kakuchi, F. Ishii, and K. Tsuda, Polymer, 37, 4853 (1996).

12. J. A. Pople and G. A. Segal, J. Chem. Phys., 44, 3289 (1966)

13. K. Nishimoto and N. Mataga, Z. Physik. Chem., 12, 335 (1957). 